\title{
IOL Power Calculation and Cataract Surgery in Eyes with previous Small Incision Lenticule Extraction
}

Nikolaus Luft ( $\sim$ nikolaus.luft@med.uni-muenchen.de )

University Eye Hospital, Ludwig-Maximilians-University

\section{Roman Lischke}

University Eye Hospital, Ludwig-Maximilians-University

Walter Sekundo

Department of Ophthalmology, Philipps University and SMILE Eyes Center, Marburg Germany

\section{Rainer Wiltfang}

SMILE Eyes Clinic, Munich

Martin Bechmann

SMILE Eyes Clinic, Munich

Thomas C. Kreutzer

University Eye Hospital, Ludwig-Maximilians-University

\section{Siegfried G. Priglinger}

University Eye Hospital, Ludwig-Maximilians-University

Martin Dirisamer

University Eye Hospital, Ludwig-Maximilians-University

\section{Research Article}

Keywords:

Posted Date: March 2nd, 2022

DOI: https://doi.org/10.21203/rs.3.rs-1370454/v1

License: (c) (1) This work is licensed under a Creative Commons Attribution 4.0 International License. Read Full License 


\section{Abstract}

Small incision lenticule extraction (SMILE), with over 5 million procedures globally performed, will challenge ophthalmologists in the foreseeable future with accurate intraocular lens power calculation in an ageing population. After more than one decade since the introduction of SMILE, only one case report of cataract surgery with IOL implantation after SMILE is present in the peer-reviewed literature. Hence, the scope of the present multicenter study was to compare the IOL power calculation accuracy in post-SMILE eyes between ray tracing and a range of empirically optimized formulae available in the ASCRS post-keratorefractive surgery IOL power online calculator. In our study of 11 post-SMILE eyes undergoing cataract surgery, ray tracing showed the smallest mean absolute error (0.40D) and yielded the largest percentage of eyes within $\pm 0.50 / \pm 1.00 \mathrm{D}(82 / 91 \%)$. The next best conventional formula was the Potvin-Hill formula with a mean absolute error of $0.66 \mathrm{D}$ and an $\pm 0.50 / \pm 1.00 \mathrm{D}$ accuracy of $45 \%$ and $73 \%$, respectively. Analyzing this first cohort of post-SMILE eyes undergoing cataract surgery and IOL implantation, ray tracing showed superior predictability in IOL power calculation over empirically optimized IOL power calculation formulae that were originally intended for use after Excimer-based keratorefractive procedures.

\section{Introduction}

Small incision lenticule extraction (SMILE), with over 5 million procedures globally performed, has evolved to one of the most popular and established keratorefractive procedures for correction of myopia and myopic astigmatism. In the foreseeable future, the number of patients with prior SMILE treatment requiring cataract surgery is expected to increase accordingly in an ageing population. Inevitably, ophthalmologists will be challenged by accurate intraocular lens (IOL) power calculations for these patients.

There are three major problems in calculating IOL power after any kind of keratorefractive surgery. The first and most significant pitfall lies in the so-called keratometric index error. ${ }^{1}$ In traditional keratometry, corneal radii are only measured for the anterior corneal curvature with the posterior corneal curvature radii being empirically extrapolated based on the assumption that the ratio between the anterior and posterior corneal curvature (A/P ratio) is constant, which is not the case after keratorefractive surgery. Secondly, some standard IOL calculation formulae tend to predict a more anterior effective lens position (formula error). Thirdly, the central zone of effective corneal power, that had been artificially treated by keratorefractive surgery, is estimated from traditional (paracentral) keratometry measurements. Therefore, the corneal power tends to be overestimated (instrument error). All these factors concordantly predispose to an underestimation in required IOL power and therefore lead to dissatisfying hyperopic residual refractive error after IOL implantation in eyes with prior myopic keratorefractive surgery. ${ }^{1}$

Several methods have been introduced to address these sources of error and to reduce refractive surprises after keratorefractive surgery. ${ }^{2-9}$ New technologies for corneal power measurements that incorporate measurements of the anterior and posterior corneal radii (e.g. total keratometry ${ }^{10}$ ) were established to enable more accurate predictions. Moreover, sophisticated IOL power calculation formulae have been developed by means of empirical optimization; some of which consider pre-keratorefractive surgery data (Masket ${ }^{11}$, Modfied-Masket or Barrett True-K formula), some do not incorporate any preoperative values (Shammas ${ }^{12}$, Barrett True-K no history, Potvin-Hill ${ }^{13}$ or Haigis-L formula ${ }^{14}$ ). Conveniently, a range of these formulae is readily accessible in the American Society of Cataract and Refractive Surgeons (ASCRS) post-keratorefractive surgery IOL power online calculator.

In addition to these empirical formulae, the purely physical ray tracing approach has demonstrated very good IOL power calculation outcomes in eyes with prior laser in situ keratomileusis (LASIK) or photorefractive keratectomy (PRK). ${ }^{15-18}$ The ray tracing method measures the true shape of the cornea after corneal refractive surgery by using the 
anterior and posterior curvature radii and asphericity of these surfaces. Moreover, the central IOL thickness, the index of refraction and the true geometrical position, as defined by the ACD (distance between the posterior corneal apex and the anterior IOL apex), are used to describe the IOL and calculate its required power accurately. The ray tracing method also obviates the need for any further historical or clinical data. ${ }^{5,15-17,19-21}$ Ray tracing has been proven to provide reliable and satisfactory results in IOL calculations not only in treatment-naïve eyes but also in eyes after LASIK and PRK. ${ }^{15,16,18,21,22}$

After more than one decade since the introduction of SMILE, only one case report of IOL calculation and implantation after SMILE is present in the peer-reviewed literature. ${ }^{23}$ However, no formula comparison was reported. Hence, there is a deficiency in postoperative refractive data to optimize existing IOL power calculation formulae for post-SMILE eyes. In addition, corneal aberrometric changes after SMILE are significantly different compared to the corneal shape changes after femtosecond laser-assisted LASIK (fs-LASIK) ${ }^{24}$, which questions the validity of formulae optimized for Excimer-based photoablative procedures (e.g. the Masket formula) in post-SMILE eyes.

Consequently, the scope of the present multicenter study was to gather the first cohort of post-SMILE patients undergoing cataract extraction with IOL implantation. In this cohort, we set out to compare the refractive prediction error of IOL power calculation between ray tracing and various empirically optimized formulae available in the ASCRS post-keratorefractive surgery IOL power online calculator.

\section{Results}

A total of 11 eyes of 7 patients [1 (14\%) female] were included with a mean follow up after SMILE of $2 \pm 1$ months (range 1 to 4 ) and a mean follow up after cataract surgery of $8 \pm 11$ months (range 1 to 38). Mean period of time between SMILE and cataract procedures were $31 \pm 16$ months (range 12 to 54). Subjects' baseline characteristics are summarized in Table 2. The mean pre- and post-SMILE manifest refraction spherical equivalent (SE) was $-5.15 \pm 1.31$ diopters ( $D$; range: -7.00 to $-3.00 \mathrm{D}$ ) and $-0.48 \pm 0.57 \mathrm{D}$ (range: -1.63 to $+0.38 \mathrm{D}$ ), respectively. The mean preoperative SE before cataract surgery was $-2.44 \pm 2.48 \mathrm{D}$ (range: -7.63 to $+0.63 \mathrm{D}$ ). One patient developed a nuclear cataract, which lead to an index myopia of $-7.63 \mathrm{D}$ of SE. After cataract surgery, the mean SE amounted to $-0.68 \pm 0.65 \mathrm{D}$ (range: -2.00 to $0.00 \mathrm{D}$ ), respectively.

The performance of the investigated IOL power calculation formulae in reference to physical ray tracing is summarized in Table 3 and visualized by boxplots (Figure 1). On average, the formulae concordantly overestimated the required IOL power. Of all investigated traditional formulae, the Potvin-Hill formula yielded the smallest ME (-0.06 $\pm 0.86 \mathrm{D}$, range -1.67 to 1.22 ) and the Shammas formula resulted in the largest IOL power overestimation with a ME of $-0.96 \pm 1.14 \mathrm{D}$ (range -2.32 to 1.07 ). Ray tracing was the only method resulting in a hyperopic ME of $0.18 \mathrm{D} \pm 0.48 \mathrm{D}$ (range -0.43 to 1.22); even though in absolute terms the ME was the lowest (Table 3). Nevertheless, Kruskal-Wallis testing revealed no statistically significant differences in ME between the different IOL power calculation methods ( $p=$ 0.16).

With respect to MAE and MedAE, ray tracing achieved the smallest MAE (0.40D) and MedAE (0.36D) of all examined methods. Of the various tested formulae from the ASCRS calculator, the Potvin-Hill formula yield the smallest MAE (0.66D), closely followed by the Barrett True-K (0.80D) and the Masket formula (0.81D). The Potvin-Hill formula also yielded the smallest MedAE (0.52D) of all conventional IOL formulae. Kruskal-Wallis testing, however, revealed no statistically significant differences in MAE ( $p=0.085)$ and on MedAE ( $p=0.095)$. Regarding the variance of ME, the ray tracing method showed the smallest variance $\left(0.23 D^{2}\right)$, followed by the Potvin-Hill $\left(0.74 D^{2}\right)$ and Modified Masket $\left(0.83 D^{2}\right)$ formulae. The Haigis-L formula showed the highest variance $\left(1.63 D^{2}\right)$. 
With $82 \%$, the ray tracing method yielded the highest percentage of eyes within a refractive prediction error of $\pm 0.50 \mathrm{D}$ (Fig. 2). The next best conventional formula was the Potvin-Hill formula with an $\pm 0.50 \mathrm{D}$ accuracy of $45 \%$. The Haigis-L formula showed the lowest $\pm 0.50 \mathrm{D}$ accuracy of $9 \%$. Fisher's exact test indicated significant differences between proportions of eyes with PEs of $\pm 0.50 \mathrm{D}(\mathrm{p}=0.034)$. Bonferroni correction was employed to investigate these differences in detail, showing statistically significant differences between the ray tracing method and each of the conventional IOL power calculation formulae (all with $p<0.001$ ). No statistically significant differences could be found in the proportions of eyes with PEs of $\pm 1.00 \mathrm{D}(p=0.754)$. Nevertheless, the ray tracing method achieved the highest $\pm 1.00 \mathrm{D}$ accuracy (91\%), followed by the Potvin-Hill, Barrett True-K and Barrett True-K no history formulae (all $73 \%)$. The Shammas formula showed the lowest $\pm 1.00 \mathrm{D}$ accuracy of $55 \%$.

\section{Discussion}

In this first study of its kind, ray tracing was compared to six established IOL power calculation formulae available in the ASCRS online calculator in post-SMILE eyes undergoing cataract surgery. In our analysis, the ray tracing method showed the most accurate IOL power calculation with a ME of $0.18 \pm 0.48 \mathrm{D}$ and $82 \%$ of eyes within $\pm 0.50 \mathrm{D}$ and $91 \%$ of eyes within $\pm 1.00 \mathrm{D}$.

Our findings endorse previous, purely theoretical studies (with no actually performed cataract surgery) in eyes after SMILE. Lazaridis et al. ${ }^{25}$ used a theoretical model including virtual IOL implantation to evaluate prediction errors between ray tracing and four conventional IOL power calculation formulae. In their analysis, ray tracing yielded the smallest ME of $-0.06 \pm 0.40 \mathrm{D}$ and a PE of $\pm 0.5 \mathrm{D}$ in $81.9 \%$ of eyes, which is highly coherent with our findings after actual cataract surgery. Moreover, the lowest ME variance (as an indicator of the consistency of an IOL power calculation method), was achieved by ray tracing in both studies. Interestingly, Lazaridis et al ${ }^{25}$ reported better results for the Haigis-L formula (ME of $-0.39 \pm 0.62 \mathrm{D}$ and $53.4 \%$ of eyes with PEs within $\pm 0.5 \mathrm{D}$ ) as compared to our "real world" analysis, where Haigis-L yielded the worst $\pm 0.50 \mathrm{D}$ accuracy of only $9 \%$ of all investigated formulae and a ME of $-0.81 \pm 1.28 \mathrm{D}$.

In the second previous theoretical study, our group ${ }^{26}$ compared the predicted postoperative residual refractive error of the IOL determined by ray tracing with the residual refraction of the same IOL as predicted by a range of conventional IOL power calculation formulae available in the ASCRS post-keratorefractive surgery IOL power calculator. The Masket formula showed the smallest ME $(-0.36 \pm 0.32 \mathrm{D})$ and yielded the largest percentage of eyes within $\pm 0.50 \mathrm{D}(70 \%)$ in reference to the prediction of ray tracing, which was defined as the gold standard method for the purpose of that study. Non-inferior MEs and $\pm 0.50 \mathrm{D}$ accuracies were achieved by the Barrett True $\mathrm{K}$, Barrett True $\mathrm{K}$ no history and the Potvin-Hill formula. ${ }^{26}$

In the third purely theoretical study, Zhu et al. ${ }^{27}$ used the concept of equivalent IOL power differences (EILD) as an indicator for the "stability" of four conventional IOL calculation formulae in post-SMILE eyes. The Barrett True-K and Haigis formulae showed similar stability in eyes with axial lengths between 24 and $26 \mathrm{~mm}(85.19 \%$ vs. $88.89 \%$ for a margin of error within $0.5 \mathrm{D} ; 100 \%$ vs. $100 \%$ for a margin of error within $1.0 \mathrm{D}$ ). In eyes with axial length of $>26 \mathrm{~mm}$, the Barrett True-K formula was the most "stable" formula with respective percentages of $81.49 \%$ and $92.59 \%$ for margin errors within $0.5 \mathrm{D}$ and $1.0 \mathrm{D}$, respectively.

These compiled theoretical data is confirmed by the present "real world" study, in which the Potvin-Hill and Masket formula showed the best PEs of all conventional formulae. The Potvin-Hill formula yielded the best ME in the present study (ME $-0.06 \pm 0.86 \mathrm{D}$ and $45 \%$ of eyes within $\pm 0.50 \mathrm{D}$ ) closely followed by ray tracing (ME $0.18 \pm 0.48 \mathrm{D}$ and $82 \%$ of eyes within $\pm 0.50 \mathrm{D})$ and the Masket formula (ME $-0.25 \pm 0.98 \mathrm{D}$ and $36 \%$ of eyes within $\pm 0.50 \mathrm{D})$. Moreover, the 
accuracy of the Barrett True $\mathrm{K}$ formula was non-inferior when preoperative refractive data were not entered but estimated with the Barrett True K no history formula. By using adjusted keratometry readings, the Shammas formula showed the greatest overestimation of IOL power of all investigated formulae. Highly congruent findings were also made in the previous theoretical study of our group. ${ }^{26}$

As a purely physical approach based on Snell's law, ray tracing offers many advantages over conventional IOL power calculation formulae in post-keratorefractive surgery eyes. Unlike empirically optimized regression formulae, ray tracing does not rely on any fictional keratometric index or "fudge factors" but utilizes measurements of both the anterior and posterior corneal radii to determine total corneal power. Hence, the need for any empirical optimization, clinical history or preoperative refractive data is obsolete. The latter can be a pivotal advantage in eyes with index myopia due to cataract formation and unknown post-keratorefractive surgery refraction.

These theoretical methodological advantages of the ray tracing principle have been previously proven in different samples of post-Excimer ablation eyes undergoing cataract surgery with IOL implantation ${ }^{15,16,18}$. For instance, Savini et al. ${ }^{16}$ yielded $71.4 \%$ of 21 post myopic Excimer ablation eyes within $\pm 0.50 \mathrm{D}$ and $85.7 \%$ within $\pm 1.00 \mathrm{D}$ of the predicted refraction utilizing ray tracing. These results seem comparable to our findings in post-SMILE eyes. Saiki et al. ${ }^{18}$ reported slightly subpar outcomes for ray tracing in their sample of 24 post myopic LASIK eyes with $\pm 0.50 \mathrm{D}$ and \pm 1.00 accuracies of $42 \%$ and $75 \%$, respectively. Furthermore, the arithmetic prediction error of ray tracing of $0.63 \pm$ $0.85 \mathrm{D}$ indicated an underestimation of IOL power entailing an unpleasant hyperopic after cataract surgery. In our study, we also observed a minimal hyperopic ME for ray tracing after SMILE, even though it was more than three times smaller $(0.18 \pm 0.48 \mathrm{D})$.

First recommendations for clinicians encountering post myopic SMILE patients requiring cataract surgery can be formulated based on the findings of the present study. Physical ray tracing should be employed for IOL power calculation and surgeons should be aware of a slight hyperopic ME of less than +0.25D when selecting the appropriate IOL power, which is only available in 0.50D steps for most contemporary IOL models. Ray tracing calculations should ideally be interpreted in conjunction with the Potvin-Hill and Masket formula, which should provide comparable results.

Limitations to this study might be found. First and foremost, the study is limited by its relatively small sample size. Nevertheless, the present work represents the first cohort of post-SMILE patients undergoing cataract surgery and may provide clinicians important guidance for IOL power selection. The paucity of post-SMILE cataract cases in Austria and Germany, where the SMILE technique was developed and first introduced more than a decade ago, also prompted us to include both eyes of some patients into the analysis. For the same reason and due to the multicenter approach, the authors felt inclined to accept multiple IOL types, surgeons and surgical protocols. A further limitation of the present study is that not all formulae currently available in the ASCRS calculator could be included as no Atlas-, Galileior OCT-based corneal measurements were available.

In summary, this study comprises the first cohort of post myopic SMILE eyes undergoing cataract surgery and IOL implantation. In post-SMILE eyes, ray tracing facilitated IOL power calculation with superior accuracy and should be the first choice over conventional IOL power calculation formulae that are empirically optimized for post-Excimer ablation eyes.

\section{Materials And Methods}


This multicenter cross-sectional study included patients that had previously undergone small incision lenticule extraction (SMILE) for treatment of myopia and/or myopic astigmatism and later underwent cataract surgery with IOL implantation. The study was conducted at the University Eye Hospital of the Ludwig-Maximilians-University (Munich, Germany), the SMILE Eyes Clinic Munich Airport (Germany) the SMILE Eyes Clinic Trier (Germany), the SMILE Eyes Center at the Department of Ophthalmology of the University of Marburg (Germany) and the SMILE Eyes Clinic Linz (Austria).

Institutional review board approval, of the Ludwig-Maximilians-University Munich, was obtained for all aspects of this study; informed consent to use their data for analysis and publication was obtained from all subjects and all studyrelated procedures adhered to the tenets outlined in the Declaration of Helsinki.

\section{SMILE Surgery}

All SMILE procedures were performed by highly experienced corneal surgeons utilizing the VisuMax 500-kHz femtosecond laser platform (Carl Zeiss Meditec AG, Jena, Germany) according to the local standards of the participating centers. Surgical principles of the SMILE technique have been previously described in detail. ${ }^{28}$

\section{Cataract Surgery and IOL Implantation}

Cataract surgery including IOL selection and implantation was performed by highly experienced corneal surgeons according to the local standards of the participating centers. The implanted IOL models and powers are summarized in Table 1.

\section{Subjective Refraction}

Subjective manifest refraction was measured using the Jackson cross-cylinder method before and after each procedure. Best-corrected distance visual acuity (CDVA) was determined using standard ETDRS charts at 4 meters.

\section{Post-hoc IOL power calculation}

Post-hoc IOL power calculation was performed utilizing dedicated ray tracing software (Okulix; Panopsis, Mainz, Germany, Version 9.01) based on preoperative corneal tomography scans (Pentacam HR; Oculus Optikgeräte GmbH, Wetzlar, Germany), preoperative optical biometry and anterior chamber depth measurements (IOLMaster 500 or 700 ; Carl Zeiss Meditec AG, Jena, Germany). Moreover, the American Society of Cataract and Refractive Surgery (ASCRS) post-keratorefractive surgery IOL power online calculator (Version 4.9; http://iolcalc.ascrs.org; last accessed 04/10/2021) was used to calculate the predicted residual refractive error using the following formulae that consider pre-keratorefractive data: Barrett True K, Masket ${ }^{11}$, Modified Masket. Additionally, the following formulae available in the ASCRS calculator were used, which do not incorporate preoperative data: Barrett True K No History, Haigis- $\mathrm{L}^{14}$, Potvin-Hill ${ }^{13}$ and Shammas ${ }^{12}$. When appropriate, optimized IOL constants were used as published on the User Group for Laser Interference Biometry (ULIB) website (http://ocusoft.de/ulib/index.htm; last accessed 04/10/2021).

\section{Statistical Analysis}


On the basis of established protocols for studies on IOL power calculation formula accuracy, ${ }^{29,30}$ the prediction error (PE) was defined as the difference between the actual residual refraction and the residual refraction predicted by the respective IOL power calculation method for the same IOL power and model. The arithmetic mean of the PE was referred to as the mean error (ME). Moreover, all negative errors were converted to positive to calculate the mean absolute error (MAE) as well as the median absolute error (MedAE). Furthermore, the standard deviation, minimum and maximum (range of PE) as well as the percentage of eyes within $\pm 0.50, \pm 1.00, \pm 1.50$ and \pm 2.00 diopter (D) were reported. ${ }^{29,30}$ Boxplots were created to illustrate the differences in PE between different IOL power calculation formulae. Normal distribution was tested by the Shapiro-Wilk method. The Kruskal-Wallis test was employed to assess the differences in PE between formulae and ray tracing. In addition, the variance of ME was calculated - a smaller variance indicates better consistency of a IOL calculation method ${ }^{31}$. Fisher's exact test with Bonferroni correction was used to test for statistically significant differences between proportions of eyes with PEs within $\pm 0.50 \mathrm{D}$ and $\pm 1.00 \mathrm{D}$, respectively., A p-value of $<0.05$ was defined as indicative of statistical significance. All statistical analyses were performed using SPSS 27.0.0.0 for Windows (IBM Corp.; Armonk, NY, USA).

\section{Declarations}

\section{Data availability}

The datasets used and analyzed during the current study are available from the corresponding author on reasonable request.

\section{Author contributions statement}

Conception or design of the work: RL, NL

Acquisition, analysis, or interpretation of data: RL, WS, RW, MB, TCK, SGP, MD, NL

Drafting of the work or substantively revised: $\mathrm{RL}, \mathrm{NL}$

Approval of submission: RL, WS, RW, MB, TCK, SGP, MD, NL

Agree both to be personally accountable for the author's own contributions and to ensure that questions related to the accuracy or integrity of any part of the work, even ones in which the author was not personally involved, are appropriately investigated, resolved, and the resolution documented in the literature: RL, WS, RW, MB, TCK, SGP, MD, $\mathrm{NL}$

\section{Competing interests}

The authors have no proprietary or financial or any other competing interest in any of the materials or equipment mentioned in this study.

\section{References}

1. Hoffer KJ. Intraocular lens power calculation after previous laser refractive surgery. J Cataract Refract Surg. 2009;35(4):759-765. 
2. Wang L, Booth MA, Koch DD. Comparison of intraocular lens power calculation methods in eyes that have undergone LASIK. Ophthalmology. 2004;111(10):1825-1831.

3. Mackool RJ, Ko W, Mackool R. Intraocular lens power calculation after laser in situ keratomileusis: Aphakic refraction technique. J Cataract Refract Surg. 2006;32(3):435-437.

4. Borasio E, Stevens J, Smith GT. Estimation of true corneal power after keratorefractive surgery in eyes requiring cataract surgery: BESSt formula. J Cataract Refract Surg. 2006;32(12):2004-2014.

5. Jin H, Rabsilber T, Ehmer A, et al. Comparison of ray-tracing method and thin-lens formula in intraocular lens power calculations. J Cataract Refract Surg. 2009;35(4):650-662.

6. Abulafia A, Hill WE, Koch DD, Wang L, Barrett GD. Accuracy of the Barrett True-K formula for intraocular lens power prediction after laser in situ keratomileusis or photorefractive keratectomy for myopia. J Cataract Refract Surg. 2016;42(3):363-369.

7. Wang L, Tang M, Huang D, Weikert MP, Koch DD. Comparison of Newer Intraocular Lens Power Calculation Methods for Eyes after Corneal Refractive Surgery. Ophthalmology. 2015;122(12):2443-2449.

8. Fram NR, Masket S, Wang L. Comparison of Intraoperative Aberrometry, OCT-Based IOL Formula, Haigis-L, and Masket Formulae for IOL Power Calculation after Laser Vision Correction. Ophthalmology. 2015;122(6):10961101.

9. Canto AP, Chhadva P, Cabot F, et al. Comparison of IOL power calculation methods and intraoperative wavefront aberrometer in eyes after refractive surgery. J Refract Surg. 2013;29(7):484-489.

10. Lischke R, Mayer WJ, Feucht N, et al. Total keratometry for determination of true corneal power after myopic small-incision lenticule extraction. J Cataract Refract Surg. 2021;47(10):1285-1289.

11. Masket S, Masket SE. Simple regression formula for intraocular lens power adjustment in eyes requiring cataract surgery after excimer laser photoablation. J Cataract Refract Surg. 2006;32(3):430-434.

12. Shammas HJ, Shammas MC. No-history method of intraocular lens power calculation for cataract surgery after myopic laser in situ keratomileusis. J Cataract Refract Surg. 2007;33(1):31-36.

13. Potvin R, Hill W. New algorithm for intraocular lens power calculations after myopic laser in situ keratomileusis based on rotating Scheimpflug camera data. J Cataract Refract Surg, 2015. 41(2): p. 339-47

14. Haigis W. Intraocular lens calculation after refractive surgery for myopia: Haigis-L formula. J Cataract Refract Surg. 2008;34(10):1658-1663.

15. Rabsilber TM, Reuland AJ, Holzer MP, Auffarth GU. Intraocular lens power calculation using ray tracing following excimer laser surgery. Eye (Lond). 2007;21(6):697-701.

16. Savini G, Bedei A, Barboni P, Ducoli P, Hoffer KJ. Intraocular lens power calculation by ray-tracing after myopic excimer laser surgery. Am J Ophthalmol. 2014;157(1):150-153 e151.

17. Savini G, Calossi A, Camellin M, Carones F, Fantozzi M, Hoffer KJ. Corneal ray tracing versus simulated keratometry for estimating corneal power changes after excimer laser surgery. J Cataract Refract Surg. 2014;40(7):1109-1115.

18. Saiki M, Negishi K, Kato N, Torii H, Dogru M, Tsubota K. Ray tracing software for intraocular lens power calculation after corneal excimer laser surgery. Jpn J Ophthalmol. 2014;58(3):276-281.

19. Preussner PR, Wahl J, Lahdo H, Dick B, Findl O. Ray tracing for intraocular lens calculation. J Cataract Refract Surg. 2002;28(8):1412-1419.

20. Canovas $C$, van der Mooren M, Rosén R, et al. Effect of the equivalent refractive index on intraocular lens power prediction with ray tracing after myopic laser in situ keratomileusis. J Cataract Refract Surg. 2015;41(5):10301037. 
21. Hoffmann P, Wahl J, Preussner PR. Accuracy of intraocular lens calculation with ray tracing. J Refract Surg. 2012;28(9):650-655.

22. Ghoreyshi M, Khalilian A, Peyman M, Mohammadinia M, Peyman A. Comparison of OKULIX ray-tracing software with SRK-T and Hoffer-Q formula in intraocular lens power calculation. J Curr Ophthalmol. 2018;30(1):63-67.

23. Ganesh S, Brar S, Sriprakash K. Post-small incision lenticule extraction phacoemulsification with multifocal IOL implantation: A case report. Indian J Ophthalmol. 2019;67(8):1353-1356.

24. Gyldenkerne A, Ivarsen A, Hjortdal J. Comparison of corneal shape changes and aberrations induced By FS-LASIK and SMILE for myopia. J Refract Surg. 2015;31(4):223-229.

25. Lazaridis A, Schraml F, Preussner PR, Sekundo W. Predictability of intraocular lens power calculation after smallincision lenticule extraction for myopia. J Cataract Refract Surg. 2021;47(3):304-310.

26. Luft N, Siedlecki J, Schworm B, et al. Intraocular Lens Power Calculation after Small Incision Lenticule Extraction. Sci Rep. 2020;10(1):5982.

27. Zhu W, Zhang FJ, Li Y, Song YZ. Stability of the Barrett True-K formula for intraocular lens power calculation after SMILE in Chinese myopic eyes. Int J Ophthalmol. 2020;13(4):560-566.

28. Sekundo W, Kunert KS, Blum M. Small incision corneal refractive surgery using the small incision lenticule extraction (SMILE) procedure for the correction of myopia and myopic astigmatism: results of a 6 month prospective study. Br J Ophthalmol. 2011;95(3):335-339.

29. Hoffer KJ, Aramberri J, Haigis W, et al. Protocols for studies of intraocular lens formula accuracy. Am J Ophthalmol. 2015;160(3):403-405 e401.

30. Hoffer KJ, Savini G. Update on Intraocular Lens Power Calculation Study Protocols: The Better Way to Design and Report Clinical Trials. Ophthalmology. 2020.

31. Patel P, Ashena Z, Vasavada V, et al. Comparison of intraocular lens calculation methods after myopic laserassisted in situ keratomileusis and radial keratotomy without prior refractive data. Br J Ophthalmol. 2020.

\section{Tables}

\section{Table 1}

\section{Implanted IOL models and powers.}




\begin{tabular}{|c|c|c|c|}
\hline $\begin{array}{l}\text { Eye } \\
\text { ID }\end{array}$ & Implanted IOL Model & Manufacturer & $\begin{array}{l}\text { IOL Power (spherical equivalent, } \\
\text { Diopters) }\end{array}$ \\
\hline 1 & CT Lucia 601PY & Carl Zeiss Meditec AG (Jena, Germany) & 18.5 \\
\hline 2 & CT Lucia 601PY & Carl Zeiss Meditec AG (Jena, Germany) & 16.5 \\
\hline 3 & CT Lucia 601PY & Carl Zeiss Meditec AG (Jena, Germany) & 21.0 \\
\hline 4 & $\begin{array}{l}\text { AcrySof IQ Toric } \\
\text { SN6AT2/3 }\end{array}$ & $\begin{array}{l}\text { Alcon GmbH } \\
\text { (Freiburg, Swiss) }\end{array}$ & 25.0 \\
\hline 5 & $\begin{array}{l}\text { AcrySof IQ Toric } \\
\text { SN6AT2/3 }\end{array}$ & $\begin{array}{l}\text { Alcon GmbH } \\
\text { (Freiburg, Swiss) }\end{array}$ & 24.75 \\
\hline 6 & $\begin{array}{l}\text { Lentis Comfort LS-313 } \\
\text { MF15 }\end{array}$ & $\begin{array}{l}\text { Oculentis GmbH } \\
\text { (Berlin, Germany) }\end{array}$ & 21.0 \\
\hline 7 & $\begin{array}{l}\text { Lentis Comfort LS-313 } \\
\text { MF15 }\end{array}$ & $\begin{array}{l}\text { Oculentis GmbH } \\
\text { (Berlin, Germany) }\end{array}$ & 19.0 \\
\hline 8 & Polylens Y 50 P & $\begin{array}{l}\text { Polytech-Domilens GmbH (Roßdorf, } \\
\text { Germany) }\end{array}$ & 19.5 \\
\hline 9 & Polylens Y 50 P & $\begin{array}{l}\text { Polytech-Domilens GmbH (Roßdorf, } \\
\text { Germany) }\end{array}$ & 18.5 \\
\hline 10 & CT Asphina 409 MP & Carl Zeiss Meditec AG (Jena, Germany) & 22.0 \\
\hline 11 & CT Lucia 611 PY & Carl Zeiss Meditec AG (Jena, Germany) & 22.5 \\
\hline
\end{tabular}

$\mathrm{IOL}$, intraocular lens; $\mathrm{D}$, diopter.

Table 2

Subjects' characteristics 


\begin{tabular}{|c|c|c|c|c|c|}
\hline \multicolumn{2}{|l|}{ Parameter } & Mean & Median & SD & Range \\
\hline \multicolumn{2}{|c|}{ Age at SMILE (years) } & 46.43 & 46 & 6.75 & 37 to 55 \\
\hline \multicolumn{2}{|c|}{ Age at cataract surgery (years) } & 49.45 & 49 & 7.31 & 38 to 59 \\
\hline \multirow[t]{8}{*}{ SMILE } & \multicolumn{4}{|l|}{ Preoperative Manifest Refraction (D) } & \\
\hline & Sphere & -4.86 & -5.25 & 1.30 & -6.50 to -2.75 \\
\hline & Cylinder & -0.57 & -0.50 & 0.23 & -1.00 to -0.25 \\
\hline & Spherical Equivalent & -5.15 & -5.38 & 1.31 & -7.00 to -3.00 \\
\hline & \multicolumn{4}{|l|}{ Postperative Manifest Refraction (D) } & \\
\hline & Sphere & -0.34 & -0.5 & 0.5 & -1.75 to 0.50 \\
\hline & Cylinder & -0.27 & -0.25 & 0.24 & -0.75 to 0.00 \\
\hline & Spherical Equivalent & -0.48 & -0.50 & 0.57 & -1.63 to 0.38 \\
\hline \multirow[t]{8}{*}{ Cataract surgery } & \multicolumn{5}{|l|}{ Preoperative Manifest Refraction (D) } \\
\hline & Sphere & -2.00 & -1.5 & 2.49 & -7.00 to 1.25 \\
\hline & Cylinder & -0.89 & -1.00 & 0.58 & -2.00 to -0.25 \\
\hline & Spherical Equivalent & -2.44 & -2.25 & 2.48 & -7.63 to 0.63 \\
\hline & \multicolumn{5}{|l|}{ Postperative Manifest Refraction (D) } \\
\hline & Sphere & -0.45 & 0.00 & 0.72 & -2.00 to 0.25 \\
\hline & Cylinder & -0.45 & -0.5 & 0.4 & -1.25 to 0.00 \\
\hline & Spherical Equivalent & -0.68 & -0.63 & 0.65 & -2.00 to 0.00 \\
\hline
\end{tabular}

SD, standard deviation; D, diopter; SMILE, small incision lenticle extraction; BCVA, best corrected visual acuity.

Table 3

Formula performance in comparison. 


\begin{tabular}{|c|c|c|c|c|c|c|c|c|c|c|c|}
\hline \multicolumn{2}{|c|}{ Formula } & \multicolumn{4}{|c|}{ Predicition error (D) } & \multicolumn{2}{|c|}{$\begin{array}{l}\text { Absolute Error } \\
\text { (D) }\end{array}$} & \multicolumn{4}{|c|}{$\begin{array}{l}\% \text { of eyes within PE range } \\
\text { indicated }\end{array}$} \\
\hline & & Mean & SD & Range & $\begin{array}{l}\text { Variance } \\
\left(D^{2}\right)\end{array}$ & Mean & Median & $\stackrel{ \pm}{0.5 D}$ & $\frac{ \pm}{1.0 D}$ & $\stackrel{ \pm}{1.5 D}$ & $\frac{ \pm}{2.0 \mathrm{D}}$ \\
\hline Ray tra & ing & 0.18 & 0.48 & $\begin{array}{l}-0.43 \\
\text { to } \\
1.22\end{array}$ & 0.23 & 0.4 & 0.36 & 82 & 91 & 100 & 100 \\
\hline \multirow{3}{*}{$\begin{array}{l}\text { Using } \\
\text { prior } \\
\text { data }\end{array}$} & Masket & -0.25 & 0.98 & $\begin{array}{l}-1.99 \\
t 014\end{array}$ & 0.95 & 0.81 & 0.82 & 36 & 64 & 91 & 100 \\
\hline & $\begin{array}{l}\text { Modified- } \\
\text { Masket }\end{array}$ & -0.55 & 0.91 & $\begin{array}{l}-2.23 \\
\text { to } \\
0.94\end{array}$ & 0.83 & 0.85 & 0.67 & 27 & 64 & 91 & 91 \\
\hline & $\begin{array}{l}\text { Barret } \\
\text { True-K }\end{array}$ & -0.27 & 0.98 & $\begin{array}{l}-2.32 \\
\text { to } \\
1.07\end{array}$ & 0.96 & 0.80 & 0.72 & 27 & 73 & 91 & 91 \\
\hline \multirow[t]{4}{*}{$\begin{array}{l}\text { Using } \\
\text { no } \\
\text { prior } \\
\text { data }\end{array}$} & Shammas & -0.96 & 1.14 & $\begin{array}{l}-2.53 \\
\text { to } \\
0.67\end{array}$ & 1.3 & 1.14 & 0.94 & 27 & 55 & 73 & 91 \\
\hline & Haigis-L & -0.81 & 1.28 & $\begin{array}{l}-3.76 \\
\text { to } \\
1.17\end{array}$ & 1.63 & 1.14 & 0.84 & 9 & 64 & 82 & 91 \\
\hline & Potvin-Hill & -0.06 & 0.86 & $\begin{array}{l}-1.67 \\
\text { to } \\
1.22\end{array}$ & 0.74 & 0.66 & 0.52 & 45 & 73 & 91 & 100 \\
\hline & $\begin{array}{l}\text { Barrett } \\
\text { True K no } \\
\text { history }\end{array}$ & -0.44 & 1.13 & $\begin{array}{l}-2.90 \\
\text { to } \\
1.12\end{array}$ & 1.27 & 0.93 & 0.67 & 27 & 73 & 91 & 91 \\
\hline
\end{tabular}

\section{Figures}




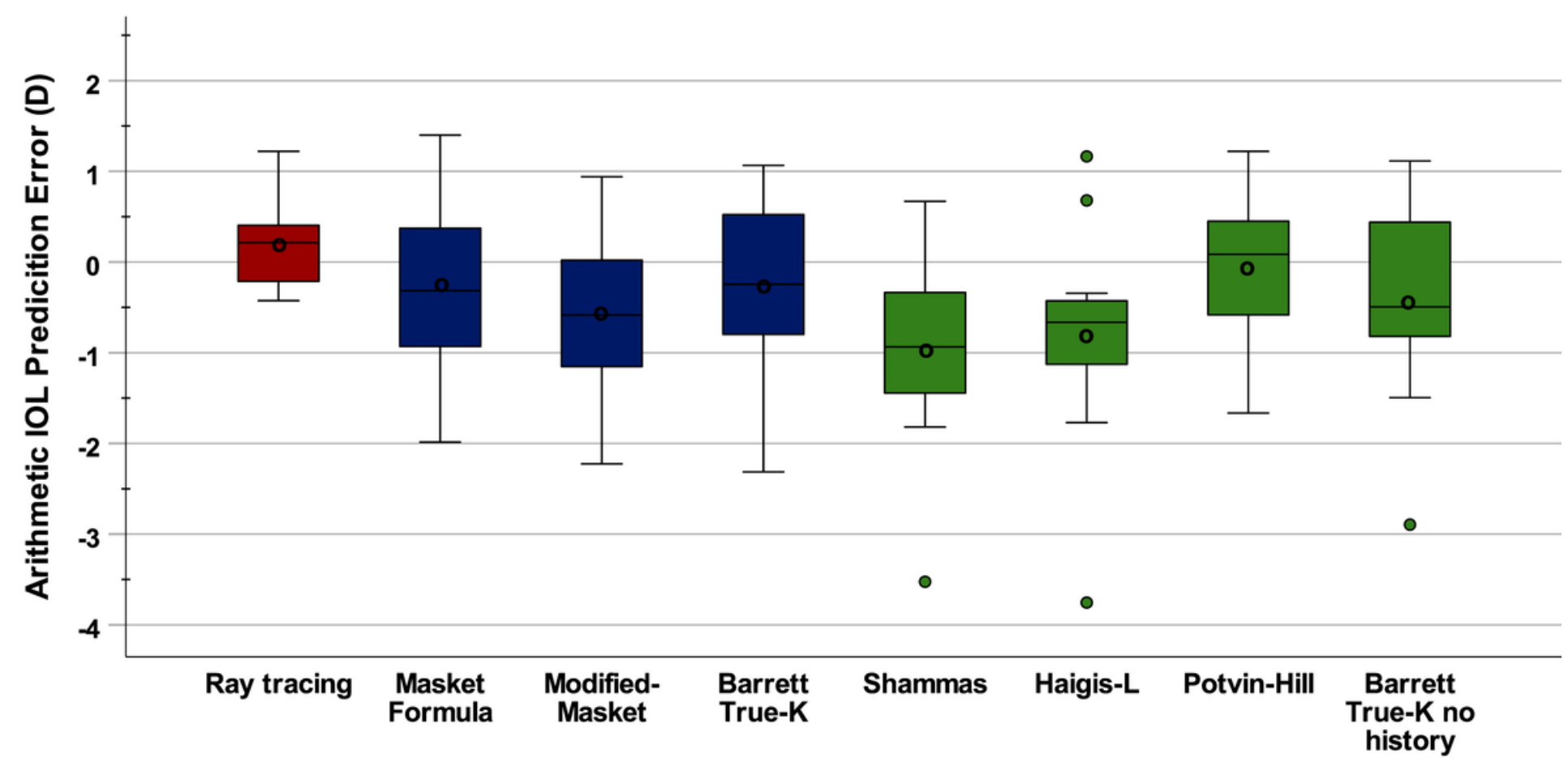

Figure 1

Prediction errors of IOL power calculation formulae.

Blue boxplots show formulae that incorporate clinical history data and green boxplots show formulae that do not use any prior-keratorefractive surgery data. The red boxplot represents ray tracing. Circles demonstrate the arithmetic means of the prediction errors. (D, diopter). 


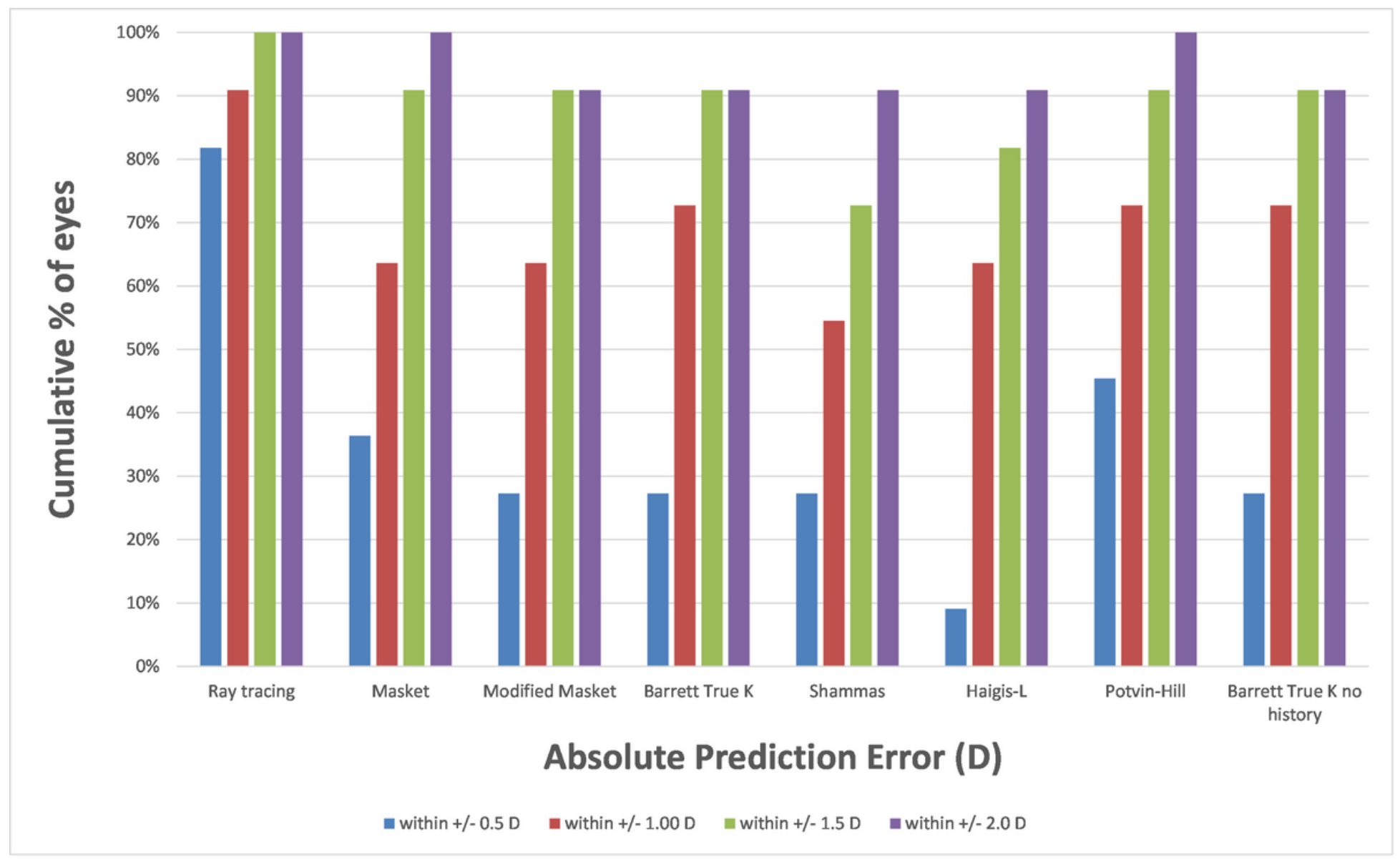

Figure 2

Histogram analysis comparing the percentage of eyes within given prediction error ranges. 